\title{
A Study on Curriculum Development Experiences of Prospective Korean Language Teachers
}

\author{
예비 한국어 교사의 교육과정 개발 경험에 대한 연구 \\ Sangsoo $\operatorname{Kim}^{1}$ \\ 김상수 1
}

${ }^{1}$ Assistant Professor, School of Interdisciplinary, Major in Korean Language Education,

Tongmyong University, Korea, kospora@naver.com

\begin{abstract}
This study started with the awareness of the problem that there is not enough opportunity for prospective Korean language teachers to experience the field of Korean language education. To address this problem, a case of 'Korean Curriculum Theory' class at P University was presented in the first semester of 2021 to show a field-oriented class case. In addition, the Korean language curriculum developed by the prospective teachers in this class, the curriculum development plan, the curriculum operation schedule, and educational materials were presented together. The Nvivo12 was used to analyze the curriculum development reflection journals written by the study participants. The subjects of the report to be analyzed were; the necessity of developing the Korean language curriculum, the difficulty of developing the curriculum, how to understand the curriculum, and what was learned through curriculum development. The results of this study is intended to provide basic data needed to develop practical educational capabilities that can be used in the educational field, breaking away from the theory-centered classes that are pointed out as a problem in the Korean language teacher education curriculum. Based on this study, it is expected that field-oriented case studies will be continuously reported so that Korean language teachers with expertise and field application skills can be cultivated.
\end{abstract}

Keywords: Korean Language Education, Curriculum Development, Educational Materials, Prospective Teachers, Korean Language Teacher Development

요약: 이 연구는 예비 한국어 교사들에게 한국어 교육 현장을 경험할 수 있는 기회가 부족하다는 문제의식에서 시작하였다. 이러한 문제를 해결하기 위하여 현장 중심의 수업 사례를 제시하고자 2021 년 1 학기에 P 대학교에서 진행한 한국어 교육과정론 수업의 운영 과정을 보고하였다. 또한 이 수업에서 예비 교사들이 개발한 한국어 교육과정과 교육과정 개발 계획서, 교육과정 운영 일정, 교육자료 등을 함께 제시하였다. 그리고 Nvivo12 를 사용하여 연구 참여자들이 작성한 교육과정 개발 성찰 보고서를 분석하였다. 보고서의 주제는 한국어 교육과정 개발의 필요성, 교육과정 개발의 어려움, 교육과정을 이해하는 방법, 교육과정 개발을 통하여 배운 것으로 그 주제 영역을 분석해 볼 수 있었다. 이 연구의 결과는 한국어 교사 교육과정에서 문제로 지적되고 있는 이론 중심의 수업에서 벗어나서, 교육 현장에서 활용할 수 있는 실제적인 교육 역량을 기르는 데 필요한 기초 자료가 될 것이다. 이 연구를 바탕으로 현장 중심의 사례 연구가 지속해서 보고되어서 전문성과 현장 적용 능력을

Received: August 05, 2021; $1^{\text {st }}$ Review Result: September 22, 2021; $2^{\text {nd }}$ Review Result: November 13, 2021 Accepted: December 31, 2021 
두루 갖춘 한국어 교사를 양성할 수 있기를 기대한다.

핵심어: 한국어 교육, 교육과정 개발, 교육자료, 예비 교사, 한국어 교사 양성

\section{1. 서론}

이 연구는 예비 한국어 교사가 교육 현장의 주체가 되어 교육의 질적 향상에 기여할 수 있는 방안이 무엇인지를 탐색하는 데 목적이 있다. 그들이 교육과정을 개발하는 동안에 무엇을 경험하고 그 경험에 대해서 어떻게 생각하는가에 관심을 기울임으로써, 그들의 경험이 한국어 교육 현장과 교육자들에게 시사하는 바가 무엇인지를 살피고자 한다.

일반적으로 교사 양성 과정의 가장 큰 문제로 지적되는 것이 예비 교사들의 교육 현장 활용 역량이다. 대부분의 교육 과정이 이론에 집중되어 예비 교사들이 실제 교육 현장을 경험할 기회가 적으며 이는 현장 활용 능력을 기르는 데 어려움이 따른다. 한국어 교사를 양성하기 위하는 과정도 예외는 아니어서, 한국어 교사가 되기 위해 정해진 교육과정을 이수하였으나 예비 교사들이 한국어 교육 현장에 필요한 실질적인 능력을 갖추는 데에는 한계가 있다[1-3].

이러한 문제는 오랜 기간 지적되고 논의되어 왔으나 여전히 다수의 한국어 교사를 양성하는 데에만 초점을 두어 기존의 이론 중심 교육과정을 현장 중심 교육과정으로 바꾸는 데에 한계가 있다. 이러한 문제점을 보완하기 위해서는 특정 교과목에서만 현장 중심의 교육을 진행할 것이 아니라, 다양한 전공 교과목에서 예비 교사들이 실제 교사가 되어 생각하고, 현장의 문제를 해결할 수 있도록 교과목을 운영할 필요가 있다[4-6].

이에 본 연구는 '한국어 교육과정론' 수업을 운영하면서 교과의 내용을 예비 교사들에게 한국어 교육 현장을 이해하는 데 도움을 줄 수 있도록 구성하였다. 예비 교사들이 이 수업을 통하여 교육과정이 무엇인가에 대한 근본적인 질문뿐만 아니라, 한국어 교육과정을 개발하는 데에 필요한 세부 내용을 함께 탐색하면서 현장 교사의 입장에서 생각할 수 있도록 하였다. 또한 한국어 교육의 대상인 외국인 학습자의 특성과 교육 환경, 교육 자료 등 한국어 교육 현장에 필요한 다양한 사항들에 대해 생각해 볼 수 있는 기회를 제공하고자 하였다.

한국어 교육과정의 경우 국가 수준의 교육과정으로 국제통용 한국어 표준모형과 $\mathrm{KSL}$ 과정 있으며, 학교 수준의 교육과정은 한국어 교육기관에서 운영하고 있는 자체 교육과정이 있다. 국가 수준의 교육과정 개발은 연구 주관 기관과 연구 참여자들의 논의를 통하여 결정된다. 그러나 한국어 교육기관의 개별 교육과정은 해당 교육 기관의 한국어 교사가 전문성과 자율성을 발휘하여 교육과정 개발과 운영에 참여할 수 있다. 즉, 외국인 한국어 학습자의 교육적 요구와 특성을 반영하여 교육 현장에 적합성을 높이고 교육의 질적 향상에 기여할 수 있다[7][8].

한편, 한국어 교육 분야에서는 한국어교육과정과 관련된 많은 연구들이 진행되었는데, 교육과정과 숙달도 단계에 따른 교수 목표와 내용 그리고 일반 목적이나 특수 목적 등 목적별 교육과정 개발에 대해서 김정숙(2000), 안경화 외(2000), 민현식(2004), 이해영(2004), 이미혜(2008) 등에서 논의하였다[9][10][11][12][13]. 교수요목 설계와 교육과정 개발에 필요한 논의를 위해 정명숙(2003), 김인규(2003) 등에서 기초 연구가 이루어졌다[14][15]. 최근 들어 주목받는 교육과정 논의로는 김지혜 외(2018), 박진욱(2018) 
등의 국제통용 한국어교육 표준모형 연구[16][17], 강수정 외(2017), 양영희(2019), 심상민(2020), 김호정(2020) 등의 KSL 관련 연구[18-21]와 류선숙 외(2020), 최은경 외(2020), 권은영(2020), 문정현(2020) 등의 학문 목적 한국어 교육과정에 대해 많이 논의되고 있다[22-25].

이상에서 살펴본 바와 같이 그동안 이루어진 한국어 교육과정 관련 연구는 대부분 이론적인 탐색이나 교육과정 개발 그리고 교육과정 운영 등과 관련된 연구가 대부분을 차지하고 있다. 이는 한국어 교육 현장의 다양한 교육과정 운영과 이를 개선하는 데 많은 도움을 주었으며, 현재 운영되고 있는 한국어 교육과정을 안정화 시키는 데 밑바탕이 되었다. 하지만 다양한 한국어 학습자 군과 이들의 한국어 학습 목적은 차별화된 교육과정을 요구하는 경우가 많아서 한국어 교육 현장에서는 여전히 새로운 교육과정의 개발과 적용 방안을 찾고 있다.

\section{2. 연구 방법}

\section{1 수업 설계와 진행}

한국어 교육과정론 수업은 부산 소재 $\mathrm{P}$ 대학의 교육대학원 한국어교육학과 석사과정 교과목으로 개설되었다. 이 수업을 수강하는 학생들은 석사과정에 재학 중이며 한국어 교육현장 경험이 전혀 없는 예비 한국어 교사들이다. 이 교과목은 한국어교원자격증을 취득하기 위해서 개설되는 5 개 영역의 교과목 중에서 3 영역인 외국어로서의 한국어교육론 영역에 포함된다.

[표 1] 수업 개관

[Table 1] Class Overview

\begin{tabular}{|c|c|}
\hline 항목 & 내용 \\
\hline 수업 운영 & 2021년 1학기 16주 \\
\hline 교과목 & 한국어 교육과정론 \\
교과목 개설 대학 & P대학교 교육대학원 한국어교육전공 석사과정 \\
\hline \multirow{2}{*}{ 수강인원 } & 남 2 \\
& 여 13 \\
총 15 명 \\
\hline
\end{tabular}

\section{2 주차별 수업 계획}

이 수업은 전체 16 주로 진행되었는데 이론 수업 4 주와 중간고사, 기말고사 기간을 제외하고 실제 교육과정 개발은 10 주 동안 진행되었다. 연구 참여자들에게는 2 주간의 한국어 단기 교육과정을 개발하도록 요청하였으며, 학습 대상이나 교육 목표, 교육 자료 등의 사항은 팀원 간의 논의를 통하여 구성할 수 있도록 하였다. 우선 5 주 차에서 7 주 차까지는 팀원을 구성하고 교육과정 개발 계획서를 작성하도록 하였다. 교육과정 계획서를 작성하는 과정에서 교육대상을 특정하고 그들이 가지고 있는 한국어 학습자로서의 특징을 기록하도록 하였다. 또한 교육과정의 총괄 목표와 세부 목표를 
분류하고 내용을 작성하도록 하였다. 9주 차에는 교육과정 구성에 따른 교육과정 운영 표를 작성하도록 하였으며, 10 주 차에는 중간 점검을 위하여 팀별 발표를 진행하여 담당 교수자와 다른 수강생들의 피드백을 받도록 하였다. 11주 차부터 13주 차까지는 완성된 교육과정을 기반으로 한 단원의 교육자료를 개발하도록 하였다. 14주 차에는 마지막으로 각 팀에서 개발한 교육과정과 관련 결과물에 대해서 발표와 점검을 받도록 하였으며, 16 주 차에는 최종 결과물을 제출하도록 하였다.

\section{3. 연구 결과 및 논의}

\section{1 교육과정 개발 최종 결과}

교육과정 개발에 참여한 학생들은 최종 결과물로 두 가지 자료를 제출하였다. 첫 번째는 팀별 공동 결과물로써 교육과정 개발 계획서와 교육과정 개발 진행 과정표, 교육과정 운영 일정표 그리고 최종 교육과정과 교육자료이다. 두 번째는 개별 결과물로써 교육과정 개발 과정에서 느낀 자신의 소감을 작성한 교육과정 개발 과정과 경험에 대한 개인보고서이다.

[그림 1]은 교육과정 개발 계획이다. 교육과정 개발 계획서의 경우 팀원들에 대한 기본사항과 함께 각 팀에서 논의해야할 사항을 작성하도록 하였다. 논의 사항은 교육과정을 개발하는 데 있어서 교육 대상을 누구로 삼을 것인가에 대한 문제와 그 교육 대상이 어떠한 특징을 가지고 있는지를 논의하도록 하였다. 그리고 실제 교육과정을 설계하고 개발하기 하기 위해서 주의해야 할 사항이 무엇인지에 대해서 그 내용을 기록하도록 하였다. 교육대상을 특정한 후에는 그 교육대상에 맞는 한국어 교육과정의 총괄 목표와 세부 목표를 제시하도록 하였다.

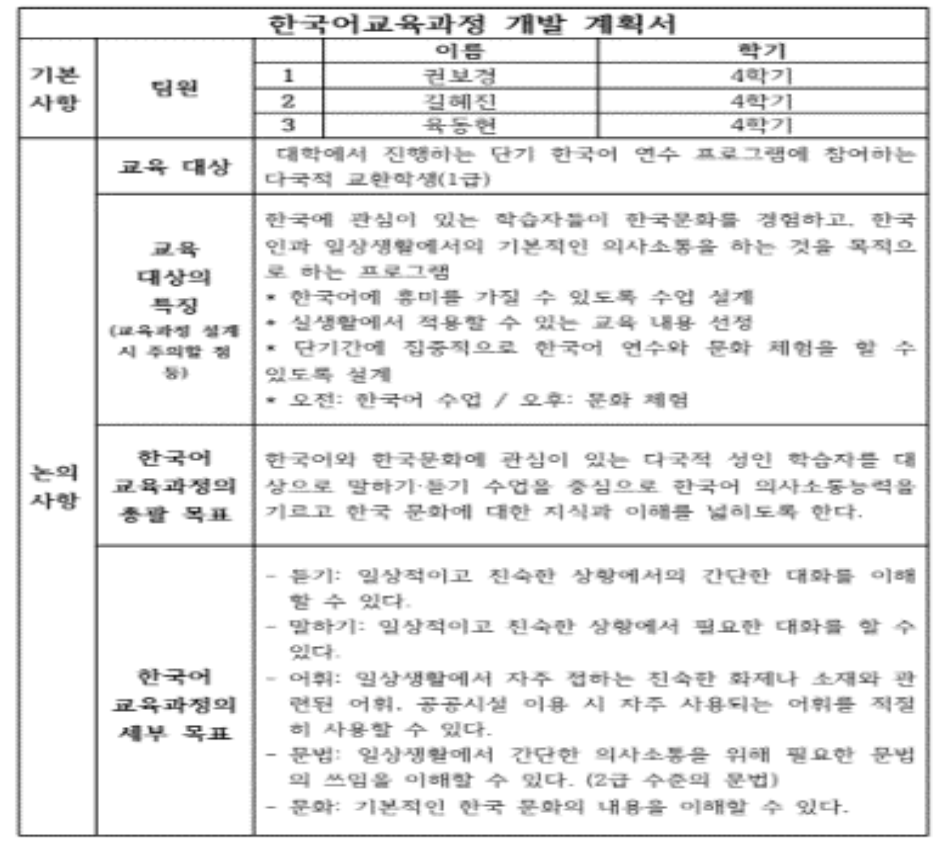

[그림 1] 교육과정 개발 계획서

[Fig. 1] Curriculum Development Plan 
[그림 2]는 교육과정 개발 진행 과정표이며, 교육과정 개발 진행 과정표는 팀원들이 교육과정을 개발하기 위하여 주어진 기간동안 함께 논의한 내용을 기록하도록 하였다. 교육과정을 개발하기 위해 팀원들과 어떠한 의견을 주고 받았으며, 최종 결과물을 산출하기 무엇을 중심으로 논의하였는지를 기록하였다. 이 과정을 통하여 교육과정을 개발하기 위해 실제로 어떠한 논의가 이루어져야 하며, 그것을 해결하기 위해 발생하는 문제점을 어떻게 처리할 것인가에 대해서 고민하게 된다. 한국어 교육 현장은 학습자 군이 더욱더 다양화되고 있으며, 그에 따라 학습 목적 또한 다양해지고 있다. 따라서 교육과정을 개발자뿐만 아니라 교육에 참여하는 모든 이들이 교육 현장의 변화와 흐름에 유연하게 대처할 수 있을 것이다.

한국어 교왁과정 개발 진행 과정

\begin{tabular}{|c|c|}
\hline 2 연 & 는의 내용 멫 결론 \\
\hline $\begin{array}{l}5 / 5 \\
(\hat{7})\end{array}$ & 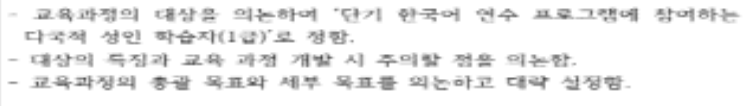 \\
\hline $\begin{array}{l}5 / 9 \\
\text { (ఖ) }\end{array}$ & 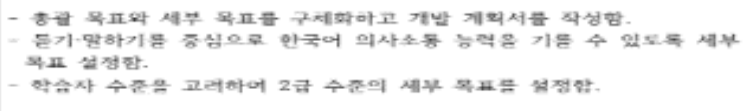 \\
\hline $\begin{array}{l}5 / 16 \\
\text { (얼) }\end{array}$ & 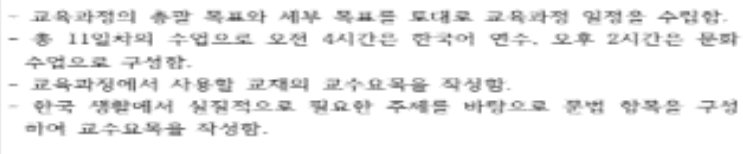 \\
\hline $\begin{array}{l}5 / 23 \\
\text { (일) }\end{array}$ & 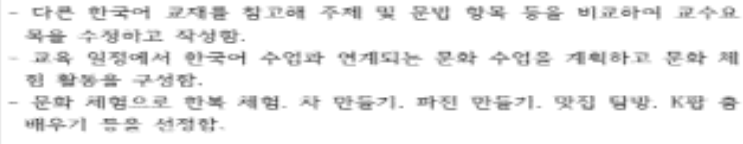 \\
\hline $\begin{array}{l}5 / 30 \\
\text { (일) }\end{array}$ & 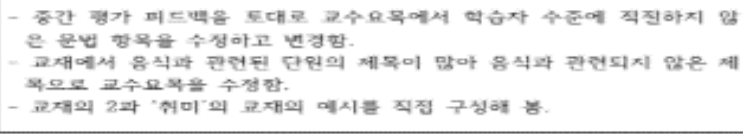 \\
\hline
\end{tabular}

[그림 2] 교육과정 개발 진행 과정

[Fig. 2] Curriculum Operation Schedule

\begin{tabular}{|c|c|c|c|}
\hline \multirow{7}{*}{$\begin{array}{l}\text { 한글 } \\
\text { 익히기 }\end{array}$} & \multicolumn{2}{|c|}{ 세부 기능 } & 한글 자음. 모음 익히기 \\
\hline & \multicolumn{2}{|c|}{ 주제 및 상황 } & 한글 익히기, 자기 이름 쎠 보기 \\
\hline & \multicolumn{2}{|c|}{ 문법 및 표현 } & 자음, 모음 \\
\hline & \multicolumn{2}{|c|}{ 어휘 } & 이릉 관련 어휘(이름. 성명, 성함) \\
\hline & \multirow{2}{*}{ 과제 } & 교실 & $\begin{array}{l}\text { 1. 나의 이름 써 보기 } \\
\text { 2. 치구 이름써 보기 }\end{array}$ \\
\hline & & 거리 & 3. 시티 투어 중 인상 깊은 곳 이름을 한글로 적어오기 \\
\hline & \multicolumn{2}{|c|}{ 문화 } & 시티 투어 버스 체험 \\
\hline \multirow{7}{*}{$\begin{array}{l}\text { 자기 } \\
\text { 소개 }\end{array}$} & \multicolumn{2}{|c|}{ 세부 기능 } & 인사하기. 소개하기 \\
\hline & \multicolumn{2}{|c|}{ 주제 및 상황 } & 인사. 소개 \\
\hline & \multicolumn{2}{|c|}{ 문법 및 표현 } & 은/는, 이에요/예요, 네/아니오 \\
\hline & \multicolumn{2}{|c|}{ 어휘 } & 국적, 직업(선생님. 학생), 인사말 \\
\hline & \multirow{2}{*}{ 곽제 } & 교실 & $\begin{array}{l}\text { 1. 반 친구들과 인사하기 } \\
\text { 2. 자신을 소개하기 }\end{array}$ \\
\hline & & $\begin{array}{l}\text { 한복 } \\
\text { 체형실 }\end{array}$ & 3. 한복 입고 한국식 인사해보기 \\
\hline & \multicolumn{2}{|c|}{ 문화 } & 한복 체험. 한국인의 인사 예절 \\
\hline
\end{tabular}

[그림 3] 교수요목

[Fig. 3] Syllabus 
[그림 3]과 [그림 4]의 자료는 교육과정 진행 일정표와 교수요목 그리고 팀에서 개발한 교육자료의 일부이다. 2주 간의 교육과정을 실제 교육현장에서 어떻게 운영할 것인지 교육과정 운영 일정표를 작성하도록 하였다. 교수요목 작성과 교육자료 개발의 경우 한국어 교육의 내용, 교수방법 등과 관련이 있기에 연구 참여자들은 기존의 한국어 교재를 참고하도록 하였다. 이 과정을 통하여 연구 참여자들은 한국어 학습 대상에 따른 교육 내용과 교육 방법 등에 대해서 확인할 수 있었다.

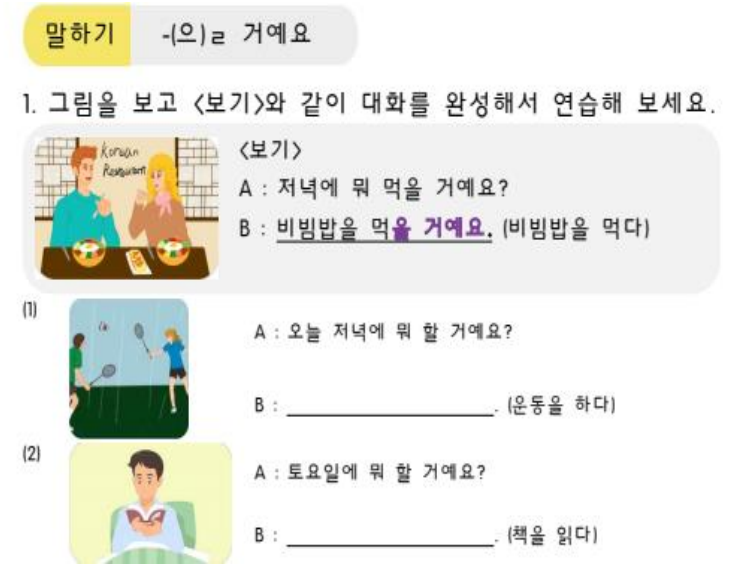

[그림 4] 교육자료

[Fig. 4] Teaching Material

\section{2 교육과정 개발 경험}

교육과정 개발 보고서는 한국어 교육과정 개발 과정에서 느낀 학습자의 소감과 성찰의 내용을 중심으로 작성하였다. 보고서 분석을 통하여 교육과정 개발 경험이 학습자들에게 어떤 영향을 끼쳤는지 살펴보았다. 연구 참여자들은 교육과정 개발에 참여하면서 경험적 성찰에 관해서 서술하였는데, 보고서의 내용은 현행 프로그램 중에서 질적 연구에 가장 최적화되어 있는 Nvivo12를 사용하여 분석하였다. 교육과정 개발 경험을 통해서 드러난 예비 교사들의 성찰 내용을 몇 개의 항목으로 분류하여 살펴보면 다음과 같다.

\subsection{1 교육과정 개발 경험의 필요성}

예비 교사이지만 교육과정 개발을 경험함으로써 이러한 경험이 왜 필요한지에 대해 알게 되었다는 내용이 많았다. 처음에는 예비 교사에게 교육과정을 개발하는 연습이 왜 필요하며, 대학원 전공과정 수업을 이렇게 운영하는 것이 어떠한 의미가 있는지 모르겠다는 연구 참여자도 있었다. 또한 이들이 실제 교육 현장에서 한국어 교사로서 업무를 시작한다고 하더라도 교육과정을 개발하는 업무를 담당하는 것을 자신들의 영역이 아니라고 생각하는 경우가 많았다. 하지만 교육과정 개발에 참여하면서 결과적으로 이 과정이 자신들에게 유익한 경험이었다고 보고하였다.

실제 교육 현장에서 학생들을 가르치는 교사가 교육 과정을 계획하는 일은 흔하지 않다. 특정 교육기관에서 직책이 높은 교사나 관리자가 교육과정을 설계하는 경우가 대부분이다. 그렇기 때문에 나도 수업을 듣기 전까지 우리에게 교육과정을 개발하는 
활동이 어떤 의미가 있는지 의문이 들었다.(P3)

대상의 선정부터 하루 일과의 시간 배분, 교수 자료 개발까지 직접 참여하여 의논하고 결정할 수 있었다. (P8)

조별 과제는 약간의 부담감으로 다가왔고 과제 역시 쉽지는 않겠지만 실제 외국인 학생들을 대상으로 교수요목을 짜고 교재까지 만들어 보는 교육적 경험은 한국어 교사로 성장하는데 좋은 밑거름은 될 듯 싶었다. (P11)

\subsection{2 교육 대상 선정과 이해의 어려움}

이들이 교육과정 개발에 참여하면서 가장 힘들었다고 보고한 내용은 교육 대상을 선정하고 그 교육 대상이 어떠한 특징을 가지고 있는지를 파악하는 일이었다. 연구 참여자들은 자신들이 교육 대상 선정과 이해에 어려움을 겪는 이유가 현장 경험이 없기 때문이라고 보고하였다. 예비 교사들이 교육 현장에 나가기 전에 교육 대상을 이해하는 방법은 이론적 배경과 간접 경험을 통해 얻을 수 있는 분산된 정보에 의존할 수밖에 없다. 따라서 이들이 전공 교육과정을 이수하는 동안 한국어 학습자를 직접 경험하고 이해할 수 있도록 제도적 장치를 마련하는 일을 반드시 필요한 부분이라고 여겨진다.

다문화 가정 중학생의 특징을 명확하게 아는 사람은 없었다. 그뿐만 아니라 다문화 가정의 중학생이라 할지라도 한국어 습득의 정도가 천차만별일 것이라는 생각이 들었기에 $\cdots$. 그 기준을 어디에 맞출지가 또 큰 고민이었다.(P10)

과제를 위해 제일 먼저 해야 했던 교육 대상 정하기부터 우리는 모두 의견이 달랐다. 그 와중에 더 힘들었던 것은 앞서 언급했듯 아무도 한국어를 가르쳐 본 경험이 없다는 점이었다.(P12)

한국어 교육 현장에서의 실전 경험을 가지고 있지 못하다는 약점을 만회하기 위해서라도 더 많은 시간과 노력을 이번 계획서 과제에 투입했어야 했다. (P15)

\subsection{3 교육과정 개발을 이해하는 방법}

연구 참여자들은 교육과정 개발이라는 과제를 수행하기 위해서 각자의 방식으로 교육과정의 개념을 이해하려고 하였다. 이들은 대부분 이론 강의를 통하여 학습한 배경지식과 자신들의 과거 외국어 학습 경험이나 현재의 직장 업무를 수행하면서 얻은 경험을 한국어 교육과정 개발에 적용하였다고 보고하였다.

직장에서 2 주 내외의 단기로 흥미 위주의 프로그램은 수년간 짜본 경험이 있지만 한국어 교육과정과는 전혀 다른 종류라 접근이 완전히 달라야 했다.(P2)

실제로 영어 어학원을 경영하면서 학생들을 인솔하여 호주, 캐나다, 미국 등 어학 캠프를 기획하고 또 직접 아이들을 이끌어 현지 훈련을 해 본 경험이 있기에, 비록 언어 대상이 달라도 언어의 본질은 같음을 알고 있었다. $(\mathrm{P} 4)$

미국인인 Joyce라는 이름의 먼 친척 아이를 떠올리며 그 아이를 이번 교육 학습자 모델로 상상하기로 했고 그 아이가 2 주 동안 한국에 다니러 와서 언어능력 향상은 물론 자랑스러운 한국인으로서의 자부심도 찾아갈 수 있는 교육과정을 만들고 싶은 욕심이 생겼다.(P13) 


\subsection{4 교육과정 개발을 성공적으로 마칠 수 있었던 원동력}

이들은 교육과정 개발에 참여하면서 수업 시간에 배운 이론이나 내용을 기억하고, 관련 자료와 논문을 다시 찾아보면서 자연스럽게 과제를 수행하고 있었다. 특히, 팀별 설득과 협동의 과정을 통하여 교육 목표와 교육 대상에 대한 이해가 어떻게 다른지를 경험하게 되었고, 학습 내용을 구성하고 교육 자료를 만드는 데에도 다양한 의견을 받아들이고 수용하는 과정을 경험한 것으로 보고되었다.

이날의 수업을 끝마치고 우리는 온라인으로 자정이 넘어갈 때까지 토의하며 모든 것을 갈아엎었다. 초기 계획 단계에서부터 시작해서 모든 것을 전면 백지화하였다.(P5)

처음에는 막막하고 도저히 할 수 없을 것 같이 느껴졌는데 팀원들과 아이디어를 모으니 방향이 잡히기 시작했다. 대상과 목표를 정하고 나니 구체적인 방안들이 머리에 그려졌다.(P7)

다음 단계인 '세부 목표' 를 짜기 전 조원들과 서로 논문을 공유하면서 필요한 정보를 찾고 모으고 정리하는 과정을 거쳐서 수업 세부 목표를 세우게 되었다.(P9)

\subsection{5 교육과정 개발 경험을 통해 배운 것}

한국어 교육과정론 수업에서 제시한 한국어 교육과정 개발이라는 과제를 수행하면 연구 참여자들에게 어떠한 점이 유익하였는지를 확인하였다. 전공 과정을 이수하면서 한국어를 외국인 학습자에게 어떻게 가르칠 것인가에만 집중하였는데, 교육과정 개발 경험을 통하여 한국어 교육이 이루어지는 교육 현장 전체를 바라볼 수 있게 되었으며, 학습자들에 필요한 것이 무엇인가에 대해서 구체적으로 고민하게 되었다는 보고내용을 확인할 수 있었다. 또한 교육과정 개발의 어려움과 자신의 주변에 있는 외국인 학습자들에 대한 관심이 중가하였음을 보고하였다.

앞으로 직접 교육과정을 만들게 될 일이 있을지는 모르겠지만, 직접 교육과정을 개발하면서 단순히 한국어를 가르치는 것만이 아니라 전체적인 교육과정을 보도록 시각을 넓힐 수 있었다.(P8))

교육과정을 개발하면서 학습자들에게 진정으로 필요한 것은 무엇인지 효과적으로 지도하기 위한 방안에는 무엇이 있을지 오랫동안 고민하였다.(P12)

이 교육과정 개발과제를 통해 현장 다문화 학생들에게 눈길이 저절로 가게 되었다. 나의 과제 주제이기도 했지만, 학교 현장에 있는 나로서는 점차 다문화 학생이 많아지는 게 피부로 와닿는다.(P13)

과제 초반의 욕심에 비해 평범한 계획서를 제출하게 된 것은 후회가 많이 남지만 이번 협업 과정으로 인해 한국어 교육과정을 짜는 것이 얼마나 많은 준비 작업과 전문가의 노력을 해야 하는 일인지 맛보기로 체험했다는 점에 의의를 두려 한다.(P15)

\section{5. 결론}

이 연구는 예비 한국어 교사들에게 한국어 교육과정 개발의 기회와 경험을 제공하기 위하여 실시한 한국어 교육과정론 수업의 사례를 자세히 보이고, 그 과정에서 학습자들이 경험한 내용을 살펴보았다. 한국어 교육과정 개발에 관한 이론적인 논의는 많이 있었으나 예비 한국어 교사를 위한 교육과정 개발 사례나 그 과정에서 예비 
교사들이 경험을 제시한 연구는 없었기에 이 연구에서 새로운 교육 사례를 제시하고자 하였다. 특히, 한국어 교육과정론 수업을 진행하면서 현장에 적용가능한 교육과정을 개발하고, 교육과정을 운영하는 데 필요한 현장 중심의 수업을 진행하였다는 데에 의의가 있다.

본 연구에서는 한국어 교육과정론 수업 설계와 진행 과정, 주차별 수업 내용과 과제 등을 보였으며, 예비 한국어 교사들이 교육과정 개발 과정에서 작성한 최종 결과물을 보여주었다. 그리고 교육과정 개발에 참여하면서 경험한 연구 참여자들의 성찰적 경험 내용을 제시하였다. 이는 한국어 교사 교육과정에서 문제로 지적되고 있는 이론 강의 위주의 수업에서 벗어나서 복잡하고 다양한 교실 현장에서 활용할 수 있는 실제적인 교육 역량을 기르는 데에 유용한 방법이 될 수 있을 것이다.

좋은 한국어 교사는 한국어에 대한 지식과 교육 기술뿐 아니라 다양한 교수 환경과 맥락 속에서 학생과 교육 관계자들의 요구를 이해하고 그에 맞는 교육을 실시할 수 있는 능력을 갖춘 사람이어야 할 것이다. 이 연구를 시작으로 한국어 교사를 양성하기 위한 전공 교육과정을 운영하면서 현장 중심의 사례 연구가 지속적으로 보고되어 전문성과 현장 적용 능력을 두루 갖춘 한국어 교사를 양성할 수 있기를 기대한다.

\section{References}

[1] H. K. Song, S. I. Yang, An analysis on teachers' perception on the education curriculum for prospective Korean language teachers, Journal of Korean Language Education (2017), Vol.28, No.1, pp.113-139. DOI: 10.18209/iakle.2017.28.1.113

[2] J. M. Ahn, A Case study of Curriculum development in Korean teaching Using Problem-Based Learning, Journal of Learner-Centered Curriculum and Instruction, (2018), Vol.18, No.18, pp.981-1002, DOI: 10.22251/jlcci.2018. 18.18 .981

[3] K. H. Ahn, M. A. Kim, A Study on Setting Goals for Curriculum Development in Korean Language Education, The Journal of Language \& Literature, (2014), Vol.59, pp381-405, DOI: 10.15565/j11.2014.09.59.381

[4] S. M. Sim, A Critical Review of the Revised Korean as a Second Language(KSL) Curriculum: A Comparison of Listening and Speaking Achievement Standards in the Australian English as an Additional Language/Direct(EAL/D) Curriculum, Journal of Speech Communication, (2020), No.47, pp.169-204, DOI: 10.18625/jsc.2020..47.169

[5] Y. S. Goo, Expert's perspectives on improving the national Korean as a second language curriculum, Korean Education, (2015), No.105, pp.247-280, DOI: 10.15734/koed..105.201512.247

[6] Y. R. Kim, A review of the national curriculum of Korean language as a second language, Korean Language Education Research, (2014), Vol.49, No.3, pp.60-92, DOI: 10.20880/kler.2014.49.3.59

[7] H. Y. Son, T. R. Cho, A Study on the Improvement of $<$ Korean Language Curriculum $>$ : Focused on Subject of Education and System of Content, Korean Education, (2015), No.104, pp.327-361, DOI: 10.15734/koed..104.201509.327

[8] H. R. Shim, S. J. Park, Theory and actuality of academic Korean within 'Korean as a second language curriculum', The Language and Culture, (2017), Vol.13, No.3, pp.97-116, DOI: 10.18842/klaces.2017.13.3.005

[9] H. S. Min, Methocology for the Description of Curriculum of Korean Language as a Foreign Language, Journal of Korean Language Education, (2004), Vol.15, No.1, pp52-92, UCI: G704-000597.2004.15.1.007

[10] J. S. Kim, Research on the Design of Korean Language Curriculum for Academic Purposes, Journal of Korean Language Education, (2000), Vol.11, No.2, pp.1-19, UCI(KEPA): I410-ECN-0102-2009-710-005828623

[11] H. Y. Lee, Research on Course Design of Korean for Academic Purposes, Journal of Korean Language Education, (2004), Vol.15, No.1, pp.137-164, UCI: G704-000597.2004.15.1.005 
[12] K. H. Ahn, J. H. Kim, E. G. Choi, Toward a Learner-Centered Approach to Curriculum Development, Journal of Korean Language Education, (2000), Vol.11, No.1, pp.67-83, UCI(KEPA): I410-ECN-0102-2009-710-006488825

[13] M. H. Lee, The Current State and Issues of Korea`s Occupational Korean Language Education, Journal of Korean Language Education, (2008), Vol.19, No.3, pp.321-347, DOI: 10.18209/iakle.2008.19.3.321

[14] M. S. Jung, A study for designing of syllabus of Business Korean, Journal of Korean Language Education, (2003), Vol.14, No.2, pp.403-421, UCI: G704-000597.2003.14.2.005

[15] I. K. Kim, Needs analysis and syllabus development of Korean for academic purposes, Journal of Korean language Education, (2003), Vol.14, No.3, pp.81-118, UCI: G704-000597.2003.14.3.005

[16] J. H. Kim, N. R. Park, H. J. Kang, Korean Language Curricula for Overseas Universities: How to Use the International Standard Curriculum of the Korean Language, Bilingual Research, (2018), No..71, pp.93-129, DOI: 10.17296/korbil.2018..71.93

[17] J. W. Park, S. M. Lee, M. J. Jang, Research on application of the Variations of Internationally circulated Standard Korean Language Curriculum, Journal of Korean Language Education, (2018), Vol.29, No.1, pp.25-52, DOI: 10.18209/iakle.2018.29.1.25

[18] S. J. Kang, J. W. Lim, Analysis on KSL Curriculum Implementation based on Muticultural Education Approaches, The Journal of Learner-Centered Curriculum and Instruction, (2017), Vol.17, No.12, pp.333-367, DOI: $10.22251 /$ jlcci.2017.17.12.333

[19] Y. H. Yang, A Suggestion on the Revision of Korean Language Curriculum as Public Education, The Journal of Learner-Centered Curriculum and Instruction, (2019), Vol.19, No.23, pp.1255-1274, DOI: 10.22251/jlcci.2019.19.23.1255

[20] S. M. Sim, A Critical Review of the Revised Korean as a Second Language(KSL) Curriculum: A Comparison of Listening and Speaking Achievement Standards in the Australian English as an Additional Language/Direct(EAL/D) Curriculum, Journal of Speech Communication, (2020), No.47, pp.169-204, DOI: 10.18625/jsc.2020..47.169

[21] H. J. Kim, Exploring the Concept and Content Structure of 'Academic Korean' within the KSL Curriculum, Multicultural Education Studies, (2020), Vol.13, No.1, pp.91-118, DOI: 10.14328/MES.2020.3.31.91

[22] E. Y. Kwon, Research for the Liberal Arts Curriculum for International Students, Korean Journal of General Education, (2013), Vol.7, No.2, pp.373-398, UCI: G704-SER000001745.2013.7.2.004

[23] S. S. Ryu, J. H. Kim, M. K Kim, M. K. Jung, A Study on the Development of Korean as a General Education Course for the Increase of Academic Ability of Foreign Undergraduates, Journal of Korean Language Education, (2020), Vol.31, No.3, pp.125-155, DOI: 10.18209/iakle.2020.31.3.125

[24] E. K. Choi, S. M. Lim, E. S. Ko, J. Y. Lim, A Study on a Customized Education Curriculum for International Students: Focusing on D University's Global Leaders Track, Journal of Dong-ak Language and Literature, (2020), No.80, pp.445470, DOI: https://doi.org/10.25150/dongak.2020..80.014

[25] J. H. Moon, Composition and Realization of Korean Culture Liberal Arts Subjects for International Students, Journal of the Korea Contents Association, (2020), Vol.20, No.2, pp.658-666, DOI: 10.5392/JKCA.2020.20.02.658 